Needle biopsy through the abdominal wall for the diagnosis of gastrointestinal stromal tumour - Does it increase the risk for tumour cell seeding and recurrence?

\author{
Eriksson, Mikael
}

2016-05

Eriksson, M, Reichardt , P , Hall , K S , Schutte , J, Cameron , S , Hohenberger , P , Bauer , S , Leinonen, M , Reichardt, A, Davis, M R , Alvegard , T \& Joensuu, H 2016 , ' Needle biopsy through the abdominal wall for the diagnosis of gastrointestinal stromal tumour - Does it increase the risk for tumour cell seeding and recurrence? ' , European Journal of Cancer , vol. 59 , pp. 128-133 . https://doi.org/10.1016/j.ejca.2016.02.021

http://hdl.handle.net/10138/223961

https://doi.org/10.1016/j.ejca.2016.02.021

publishedVersion

Downloaded from Helda, University of Helsinki institutional repository.

This is an electronic reprint of the original article.

This reprint may differ from the original in pagination and typographic detail.

Please cite the original version. 


\title{
Needle biopsy through the abdominal wall for the diagnosis of gastrointestinal stromal tumour - Does it increase the risk for tumour cell seeding and recurrence?

\author{
Mikael Eriksson ${ }^{\mathrm{a}, *}$, Peter Reichardt ${ }^{\mathrm{b}}$, Kirsten Sundby Hall ${ }^{\mathrm{c}}$, \\ Jochen Schütte $^{\mathrm{d}}$, Silke Cameron ${ }^{\mathrm{e}}$, Peter Hohenberger ${ }^{\mathrm{f}}$, Sebastian Bauer ${ }^{\mathrm{g}}$, \\ Mika Leinonen ${ }^{\mathrm{h}}$, Annette Reichardt ${ }^{\mathrm{b}}$, Maria Rejmyr Davis ${ }^{\mathrm{i}}$, \\ Thor Alvegård ${ }^{\mathrm{j}}$, Heikki Joensuu ${ }^{\mathrm{k}}$
}

\footnotetext{
${ }^{a}$ Department of Oncology, Skane University Hospital and Lund University, Lund, Sweden

${ }^{\mathrm{b}}$ Sarcoma Center Berlin-Brandenburg, HELIOS Clinic Berlin-Buch, Berlin, Germany

${ }^{\mathrm{c}}$ Department of Oncology, The Norwegian Radium Hospital, Oslo University Hospital, Oslo, Norway

${ }^{\mathrm{d}}$ Schwerpunktpraxis for Oncology, Hematology and Ambulant Tumour Therapy, Düsseldorf, Germany

${ }^{\mathrm{e}}$ Department of Gastroenterology/Endocrinology, University of Göttingen, Göttingen, Germany

${ }^{\mathrm{f}}$ Division of Surgical Oncology and Thoracic Surgery, Mannheim University Medical Center, Mannheim, Germany

${ }^{\mathrm{g}}$ Sarcoma Center, West German Cancer Center, University of Duisburg-Essen, Essen, Germany

h 4Pharma Ltd, Turku, Finland

${ }^{\mathrm{i}}$ Regional Cancer Center, Lund, Sweden

${ }^{\mathrm{j}}$ Department of Cancer Epidemiology, Lund University, Lund, Sweden

${ }^{\mathrm{k}}$ Department of Oncology, University of Helsinki, and Comprehensive Cancer Center, Helsinki University Hospital, Helsinki, Finland
}

Received 7 February 2016; accepted 22 February 2016

Available online 28 March 2016

\section{KEYWORDS}

Gastrointestinal stromal tumour; GIST;

Needle biopsy;

Imatinib;

Adjuvant;

Risk of recurrence

\begin{abstract}
Purpose: Preoperative percutaneous transabdominal wall biopsy may be considered to diagnose gastrointestinal stromal tumour (GIST) and plan preoperative treatment with tyrosine kinase inhibitors when an endoscopic biopsy is not possible. Hypothetically, a transabdominal wall biopsy might lead to cell seeding and conversion of a local GIST to a disseminated one. We investigated the influence of preoperative needle biopsy on survival outcomes. Methods: We collected the clinical data from hospital case records of the 397 patients who participated in the Scandinavian Sarcoma Group (SSG) XVIII/Arbeitsgemeinschaft Internistische Onkologie (AIO) randomised trial and who had a transabdominal fine needle and/or core needle biopsy carried out prior to study entry. The SSG XVIII/AIO trial compared 1 and 3 years of adjuvant imatinib in a patient population with a high risk of GIST recurrence
\end{abstract}

* Corresponding author: Department of Oncology, Skane University Hospital, Getingevagen 4, SE-221 85 Lund, Sweden. Tel.: +46 46177507. E-mail address: mikael.eriksson@med.lu.se (M. Eriksson). 
after macroscopically radical surgery. The primary end-point was recurrence-free survival (RFS), and the secondary end-points included overall survival (OS).

Results: A total of $47(12.0 \%)$ out of the 393 patients with data available underwent a percutaneous biopsy. No significant difference in RFS or OS was found between the patients who underwent or did not undergo a percutaneous biopsy either in the entire series or in subpopulation analyses, except for a statistically significant RFS advantage for patients who had a percutaneous biopsy and a tumour $\geq 10 \mathrm{~cm}$ in diameter.

Conclusion: A preoperative diagnostic percutaneous biopsy of a suspected GIST may not increase the risk for GIST recurrence in a patient population who receive adjuvant imatinib after the biopsy.

(c) 2016 Elsevier Ltd. All rights reserved.

\section{Introduction}

The risk of recurrence of localised gastrointestinal stromal tumour (GIST) after surgery is frequently estimated with risk factors, most commonly with tumour mitotic count, size, and site of origin (whether gastric or non-gastric) [1]. Besides these factors, tumour rupture that occurs either prior to or during surgery is also considered an established risk factor for recurrence [1]. Whether a preoperative percutaneous fine needle or core needle tumour biopsy also carries an increased risk for GIST recurrence is unknown, and this question has remained controversial.

GIST is often suspected at computerised tomography (CT) or magnetic resonance imaging (MRI) of the abdomen, but there are several other tumours that may resemble GIST in imaging and are treated differently. Lymphomas, germinal cell cancers and extraskeletal Ewing's sarcoma are examples of tumours that are primarily treated with chemotherapy. Fibromatosis (desmoid tumour), in turn, is often primarily managed with a wait-and-see strategy. Thus, an accurate preoperative histopathological diagnosis may prevent inappropriate surgery.

In selected cases preoperative imatinib treatment may reduce tumour size and facilitate surgery, especially when GIST is located in the rectum [2,3] or when gastric GIST is large in size [4]. Preoperative imatinib may allow organ sparing and preserve the gastrointestinal tract function and continuity. A tissue sample prior to starting neoadjuvant imatinib is considered mandatory for verification of the diagnosis and carrying out mutational testing, as a small proportion of GISTs harbour a mutation that renders it imatinib-insensitive [5].

An endoscopic biopsy is often preferred, but in many cases a percutaneous transabdominal wall needle biopsy is needed to obtain enough tissue material. Hypothetically, a transabdominal wall biopsy could lead to GIST seeding into the abdominal cavity and conversion of a local disease to a disseminated one, but no estimations for the size of such a risk have been presented so far.
Similarly, it is unknown whether a fine needle biopsy carries a smaller risk for seeding than a core needle biopsy, which often provides enough tissue material for mutation analysis.

International consensus guidelines on GIST are rather vague in recommending a biopsy. The guidelines both recommend or accept performing a percutaneous biopsy if an endoscopic biopsy is not feasible, but, on the other hand, warn for the potential consequences. Thus, the ESMO/European Sarcoma Network Working Group guidelines state that 'The risk of peritoneal contamination is negligible if the procedure is properly carried out. Moreover, lesions at risk in this regard (e.g. cystic masses) should be biopsied only in specialized centers' [6]. The National Comprehensive Cancer Network guidelines of the United States of America point out that 'GISTs are soft and fragile tumours. EUS-FNA biopsy of primary site is preferred over percutaneous biopsy due to the risk for hemorrhage and intra-abdominal tumour dissemination' [7].

The purpose of the present study was to compare the risk of GIST recurrence between cohorts of patients who either underwent or did not undergo a tumour needle biopsy prior to surgery. To our knowledge, the present report is the first one that evaluates the effect of a needle biopsy on the risk of GIST recurrence.

\section{Methods}

\subsection{Patients}

We studied the risk of GIST recurrence related to preoperative needle biopsy within the context of the Scandinavian Sarcoma Group (SSG) XVIII/ Arbeitsgemeinschaft Internistische Onkologie (AIO) trial that compared 1-3 years of adjuvant imatinib after radical surgery for high risk GIST [8]. The SSG XVIII/ AIO trial is an open-label, multicentre, randomised phase III study, where 400 patients with operable GIST were recruited from 24 hospitals located in Finland, Germany, Norway and Sweden between February 4, 2004, and September 29, 2008 (clinicaltrials.gov 
Identifier: NCT00116935). The study participants were randomly assigned centrally in a $1: 1$ ratio to receive imatinib orally $400 \mathrm{mg} / \mathrm{d}$ either for 12 or 36 months, and they were followed up with abdominal and pelvic CT or MRI performed at 6 months intervals during and after the treatment period [8]. The primary end-point was recurrence-free survival (RFS), and the secondary endpoints consisted of overall survival (OS) and treatment safety. The patients were required to have histologically diagnosed, KIT (CD 117) immunostaining-positive GIST, and to have a high estimated risk of recurrence according to the modified National Institutes of Health (NIH) Consensus Criteria [8,9], and Eastern Cooperative Oncology Group performance status $\leq 2$. Twentyfour patients had intra-abdominal metastases at the time of the diagnosis that could be excised macroscopically completely at surgery. In this trial the patients who were randomly allocated to receive 3 years of adjuvant imatinib turned out to have significantly longer RFS and OS as compared with the patients who received imatinib for 1 year after surgery [8].

All patients entered to the trial $(n=400)$ were included in the present analysis except for three patients who did not provide consent and four patients who had missing data about preoperative needle biopsy. Therefore, the current study cohort consists of 393 patients (Fig. 1).

Clinical and survival data were obtained from the SSG XVIII/AIO database, and data about transabdominal wall diagnostic fine and/or core needle biopsy were collected from the hospital case records. Patients with an endoscopic biopsy were grouped together with the patients who had no biopsy of any type in the analyses.

\subsection{Statistical methods}

Survival was assessed using the Kaplan-Meier life-table method. RFS was calculated from the date of randomisation to the date of GIST recurrence or death, whichever occurred first, censoring patients who were alive without recurrence. OS was calculated from the date of randomisation to the date of death censoring patients alive. RFS and OS were compared between patients who had a diagnostic biopsy through the abdominal wall and those who did not, and were described by hazard ratios (HR), where a value above 1.0 indicates an increased risk for the patients biopsied through the abdominal wall, and a value below $1.0 \mathrm{a}$ lower risk. The 95\% confidence intervals (CIs) and the $P$ values for the differences were estimated using a Cox proportional hazards model.

Since it is possible that a core biopsy only, unlike a fine needle biopsy, entails a risk for seeding and tumour recurrence, we also compared those patients who underwent a core needle biopsy through the abdominal wall with patients who did not have such a biopsy, excluding the patients with a transabdominal wall fine needle biopsy only from the analyses.

We also planned analyses to reduce the potential confounding effects related to the established prognostic factors such as tumour size and site, as such factors might influence the decision to perform a biopsy and might influence patient outcome also. For size, the analyses were thus done also within the tumour size categories $\leq 5 \mathrm{~cm}, 5-10 \mathrm{~cm}$ and $>10 \mathrm{~cm}$. Regarding tumour site, we assessed separately gastric, small intestine and rectal GISTs, whereas GISTs arising at other locations were too rare to carry out meaningful analyses.

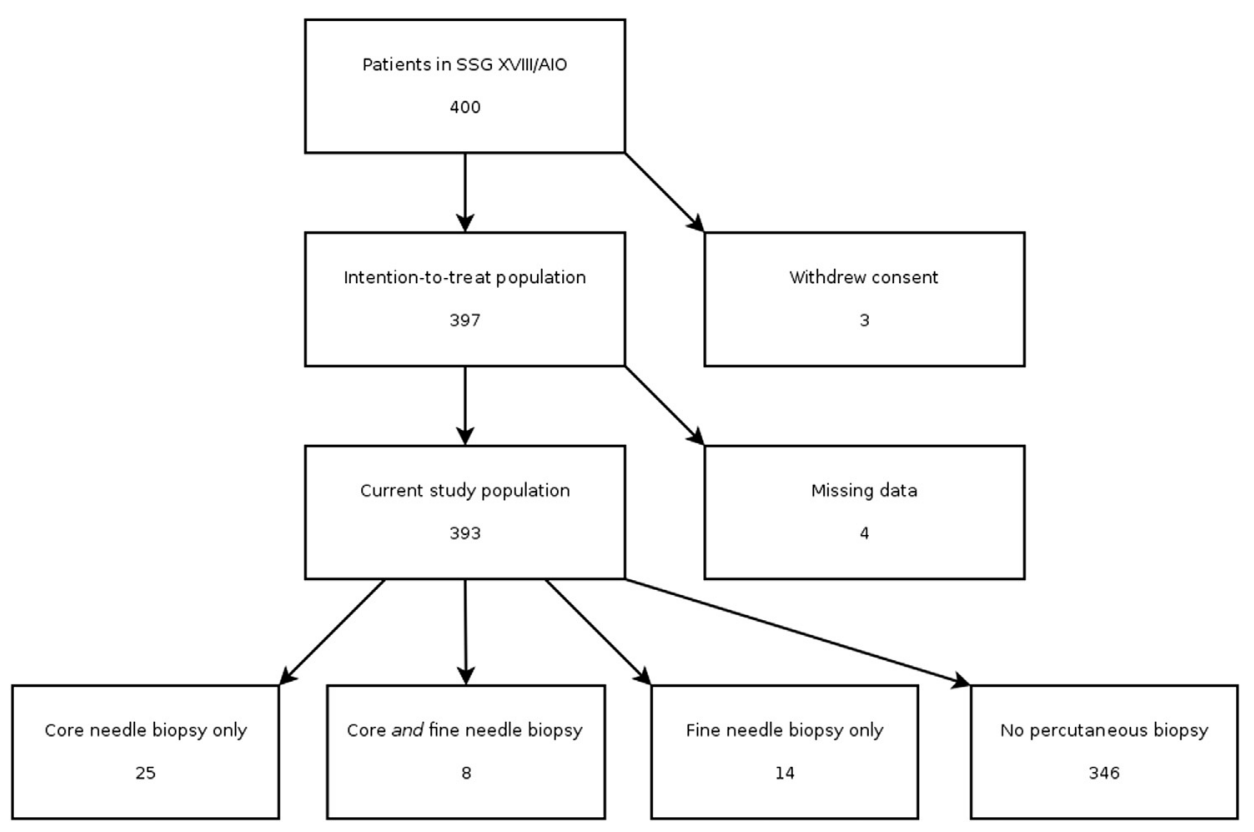

Fig. 1. A CONSORT diagram of the study. SSG, Scandinavian Sarcoma Group. 
Furthermore, separate analyses were also planned for geographical region of enrolment in the trial, as it was anticipated that the diagnostic routines could differ between study sites. Berlin was by far the single centre with the largest number of patients entered to the trial (about a fourth of all patients). Therefore, we grouped geographical areas into three categories: Berlin, other German centres, and the Nordic countries.

In addition, a stepwise multivariate analysis of RFS was performed to investigate whether the performed transabdominal wall biopsy is an independent factor when taking the established predictive factors into account. A Cox proportional hazards model with stepwise selection (entry followed by elimination) was used with the significance level of 0.05 for entry and elimination. The following factors were entered into the model as covariables: extent of disease (local or nonlocal); recurrence risk based on the NIH risk classification scheme; tumour size, site, mitotic count and rupture; GIST mutation type (KIT exon 9, KIT exon 11, any PDGFRA mutation, wild type for KIT and PDGFRA); completeness of surgery (R0 or R1); type of surgery (laparoscopic or open); age at study entry; gender; geographical region (Nordic countries, Berlin, other German sites); allocation group (adjuvant imatinib for 1 or for 3 years); and transabdominal wall biopsy (yes or no).

All $P$ values are two-sided and not adjusted for multiple testing. Statistical analyses were performed with an SAS version 9.2 for Windows (SAS Institute Inc., Cary, NC).

\section{Results}

A total of $47(12.0 \%)$ out of the 393 patients underwent a diagnostic percutaneous biopsy. Thirty-three (8.4\%) patients had a core needle biopsy, $22(5.6 \%)$ a fine needle biopsy, and eight had both (Fig. 1).

There was no significant difference in RFS between the 47 patients who did undergo a needle biopsy and the 346 patients who did not undergo any type of needle biopsy (HR 0.63, 95\% CI 0.36-1.12, $P=0.117$, Fig. 2). Similarly, there was no statistical difference in OS between these two groups (HR $0.69,95 \%$ CI $0.24-1.96$, $P=0.491$, Fig. 3). When RFS of the 33 patients who had a core needle biopsy was compared with RFS of the 346 patients who did not have any type of needle biopsy excluding the 22 patients with fine needle biopsy only from the analysis, there was no significant difference in RFS between the groups (HR $0.72,95 \%$ CI $0.38-1.38$, $P=0.323$, Fig. 4).

There was no significant difference in RFS of the patients with or without a biopsy when tumour size was $\leq 5 \mathrm{~cm}$, (HR 1.17, 95\% CI 0.15-9.03, $P=0.884$ ) or 5-10 cm, (HR 0.86, 95\% CI 0.31-2.37, $P=0.765$ ), whereas in the largest size category, $\geq 10 \mathrm{~cm}$, patients

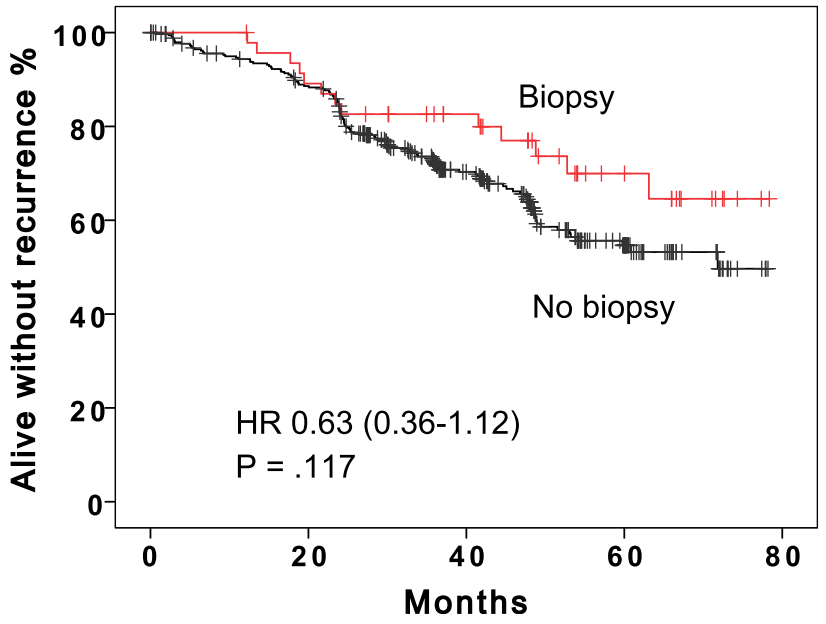

Fig. 2. Recurrence-free survival of patients who either did or did not have a diagnostic transabdominal needle biopsy performed prior to surgery and adjuvant imatinib. HR, hazard ratio.

who had a biopsy had more favourable RFS (HR 0.45, 95\% CI 0.21-0.94, $P=0.035$ ).

No statistically significant difference in RFS was found between patients who underwent a needle biopsy and those who did not among the subset of patients who had gastric GIST (HR 0.51, 95\% CI 0.18-1.44, $P=0.205$ ), those with small intestinal GIST (HR 0.85 , $95 \%$ CI $0.37-1.98, P=0.708)$, or those with rectal GIST (HR 1.62, 95\% CI 0.42-6.22, $P=0.479$ ).

Percutaneous needle biopsies were more commonly taken in the Nordic countries $(29.4 \%)$ than in Berlin $(12.8 \%)$ or the rest of Germany $(4.5 \%)$, but there were no statistically significant difference in RFS between patients who underwent a biopsy and those who did not have a biopsy when each region was considered separately.

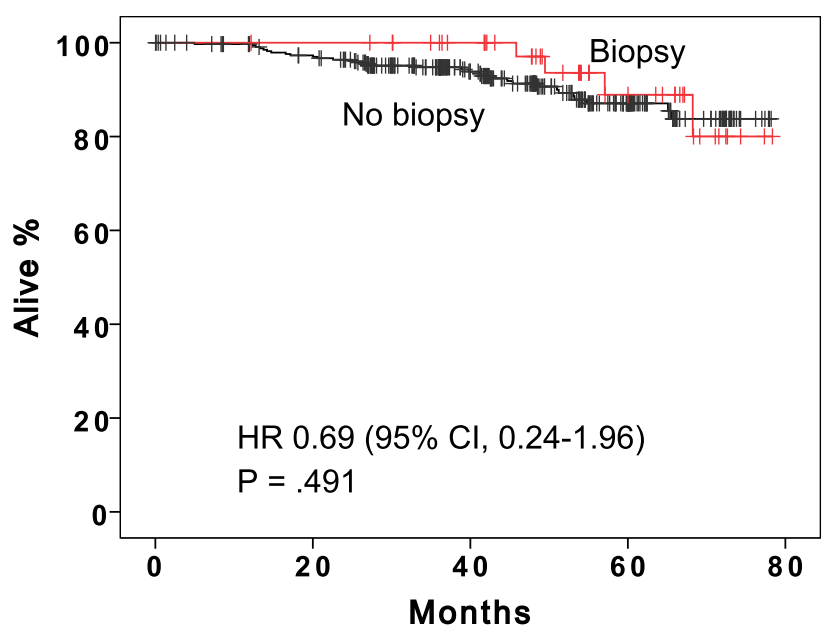

Fig. 3. Overall survival of patients who either did or did not have a diagnostic transabdominal needle biopsy performed prior to surgery and adjuvant imatinib. HR, hazard ratio. 


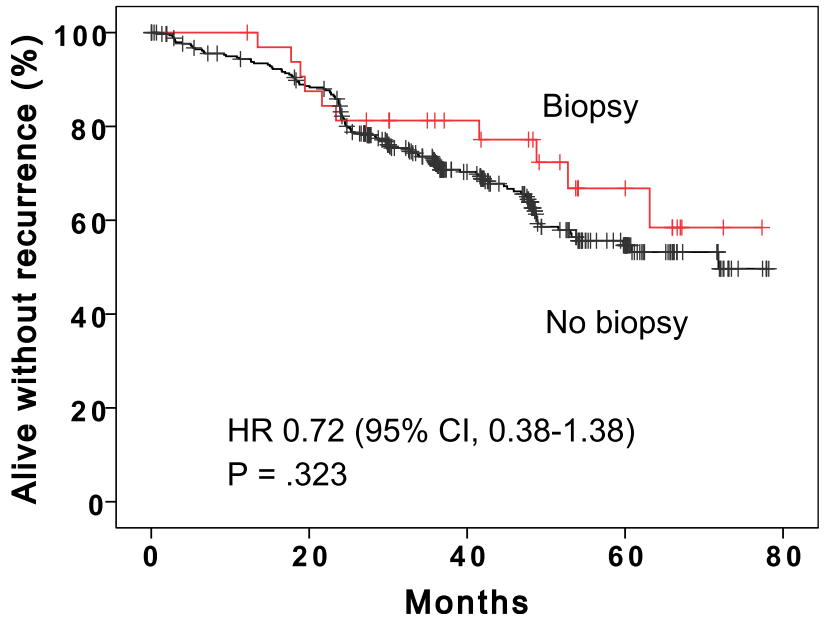

Fig. 4. Recurrence-free survival of patient who either did or did not have a diagnostic transabdominal core needle biopsy performed prior to surgery and adjuvant imatinib. HR, hazard ratio.

In a multivariable analysis only the established risk factors for GIST recurrence, a high tumour mitotic count $(P<0.001)$, non-gastric site $(P<0.001)$, presence of tumour rupture $(P<0.004)$, large tumour size, and 1year duration of adjuvant imatinib $(P<0.001)$ were independently associated with short RFS, whereas a percutaneous biopsy in history was not independently associated with RFS $(P=0.178)$.

In this study we did not focus on the potential differences between treatment arms ( 1 or 3 years of adjuvant imatinib), but on the influence of a needle biopsy on RFS. However, when RFS and OS were compared between the patients with and without a transabdominal needle biopsy in history and within each one of the two treatment arms, we found no significant differences in RFS between the patients who underwent a biopsy and those who did not in these analyses (data not shown).

\section{Discussion}

The question whether a percutaneous diagnostic biopsy increases the risk for tumour cell seeding and tumour spread has been debated for a long time, both regarding GIST and other intra-abdominal and retroperitoneal tumours including other types of sarcomas. To our knowledge, no formal studies to define this risk have been reported so far.

We collected data from the patients who had a high risk for GIST recurrence according to the modified NIH Consensus Criteria [1,9] and who participated in the SSG XVIII/AIO adjuvant trial, and compared RFS and OS between patients who underwent or did not undergo a diagnostic percutaneous biopsy prior to surgery, and also studied the key subgroups of patients for the risk of GIST recurrence. The results do not indicate an increased risk for GIST recurrence related to a percutaneous needle biopsy. Rather, there was a tendency to a decreased risk, and in the subgroup of patients who had large GIST $(>10 \mathrm{~cm})$ the difference in favour of the biopsy group reached statistical significance. Similarly, when only the patients who had a core needle biopsy performed, no harmful effect could be demonstrated.

These data must be interpreted with some caution. The study is retrospective, limited in size, and only few patients had a transabdominal wall core biopsy. The diagnostic routines likely differ between countries and centres, and many GIST physicians may refrain from a biopsy because of a feared risk of recurrence. Importantly, all patients in the current study were treated with adjuvant imatinib after the biopsy, which could have eradicated the GIST cells that leaked out from the tumour into the abdominal cavity after taking the biopsy. Therefore, the current results might not be applicable to patient populations treated with surgery alone.

The reason for the tendency towards better prognosis after pre-operative biopsies is not clear, and may be due to chance. Hypothetically, biopsies are performed more often in specialised sarcoma/GIST-centres with more experienced surgeons. A pre-operative diagnosis of GIST might make the surgeon more careful in avoiding of tumour rupture.

This study does not answer the questions about the risks for recurrence when a needle biopsy shows another type of tumour than GIST. According to the guidelines for some other types of tumours a needle biopsy should be avoided, e.g. for the adrenal gland primary tumours where even a fine needle biopsy is highly discouraged due to the risk of tumour cell spread [10]. On the other hand, some patients with another tumour type than GIST might benefit from a preoperative diagnosis, e.g. those with retroperitoneal sarcoma or lymphoma.

In conclusion, preoperative transabdominal wall needle biopsy to diagnose GIST was not associated with an increased risk for GIST recurrence in a patient population who were subsequently treated with surgery and adjuvant imatinib.

\section{Conflict of interest statement}

None declared.

\section{Acknowledgements}

The authors acknowledge the investigators and staff at all SSG XVIII/AIO institutions in Finland, Germany, Norway, and Sweden, and Mrs. Eva-Mari Olofsson and Mrs. Jeanette Ceberg at the SSG Secretariat for assistance in data collection. 


\section{References}

[1] Joensuu H. Risk stratification of patients diagnosed with gastrointestinal stromal tumor. Hum Pathol 2008:39(10):1411-9.

[2] Jakob J, Mussi C, Ronellenfitsch U, Wardelmann E, Negri T, Gronchi A, et al. Gastrointestinal stromal tumor of the rectum: results of surgical and multimodality therapy in the era of imatinib. Ann Surg Oncol 2013;20(2):586-92.

[3] Tielen R, Verhoef C, van Coevorden F, Reyners AK, van der Graaf WT, Bonenkamp JJ, et al. Surgical management of rectal gastrointestinal stromal tumors. J Surg Oncol 2013;107(4): $320-3$.

[4] Rutkowski P, Gronchi A, Hohenberger P, Bonvalot S, Schöffski P, Bauer S, et al. Neoadjuvant imatinib in locally advanced gastrointestinal stromal tumors (GIST): the EORTC STBSG experience. Ann Surg Oncol 2013;20(9):2937-43.

[5] Corless C, Schroeder A, Griffith H, Town A, McGreevey L, Harrell $\mathrm{P}$, et al. PDGFRA mutations in gastrointestinal stromal tumors: frequency, spectrum and in vitro sensitivity to imatinib. J Clin Oncol 2005;23(23):5357-64.

[6] The ESMO/European Sarcoma Network Working Group. Gastrointestinal stromal tumors: ESMO Clinical Practice Guidelines for diagnosis, treatment and follow-up. Ann Oncol 2012;23(Suppl. 7):vii49-55.

[7] NCCN Guidelines: Gastrointestinal Stromal Tumors (GIST) 2013. version 1.

[8] Joensuu H, Eriksson M, Sundby Hall K, Hartmann JT, Pink D, Schütte J, et al. One vs. three years of adjuvant imatinib for operable gastrointestinal stromal tumor. A randomized trial. JAMA 2012;307(12):1265-72.

[9] Fletcher C, Berman J, Corless C, Gorstein F, Lasota J, Longley BJ, et al. Diagnosis of gastrointestinal stromal tumors: a consensus approach. Hum Pathol 2002;33(5):459-65.

[10] Berruti A, Baudin E, Gelderblom H, Haak HR, Porpiglia F, Fassnacht M, et al. Adrenal cancer: ESMO Clinical Practice Guidelines for diagnosis, treatment and follow-up. Ann Oncol 2012;23(Suppl. 7):vii131-8. 